\title{
Robotic Mediastinal Parathyroidectomy in a Patient with Maxillary Giant Cell Tumor
}

\author{
Iskander Al-Githmi \\ Department of Surgery, Division of Cardiothoracic Surgery, King Abdulaziz University Hospital, Jeddah, KSA \\ Email: algithmi@hotmail.com
}

Received March 7, 2013; revised April 9, 2013; accepted April 18, 2013

Copyright (C) 2013 Iskander Al-Githmi. This is an open access article distributed under the Creative Commons Attribution License, which permits unrestricted use, distribution, and reproduction in any medium, provided the original work is properly cited.

\begin{abstract}
A 50-year-old woman with a maxillary giant cell tumor had primary hyperparathyroidism from a mediastinal parathyroid adenoma. Computed tomography showed a mildly enhanced, oval soft tissue nodule $(2.5 \times 1.5 \times 1.7 \mathrm{~cm})$ at the anterosuperior mediastinum. Mediastinal parathyroidectomy was performed with a 3-arm surgical robot. After surgery, the plasma parathyroid hormone level decreased markedly (before surgery, $70.5 \mathrm{pg} / \mathrm{ml}$; after surgery, $5.5 \mathrm{pg} / \mathrm{ml}$; normal reference range, 1.6 to $6.9 \mathrm{pg} / \mathrm{ml}$ ). The 3-dimensional visualization, high-dexterity, and full range of motion of the robotic system provided an efficient and safe surgical procedure for the mediastinal tumor. The purpose of this case study is to show the feasibility and effectiveness of robot assisted dissection for mediastinal parathyroid gland and to demonstrate the giant cell tumor of the bone can regress after resection of parathyroid adenoma.
\end{abstract}

Keywords: Surgery/Incisions/Exposure/Techniques; Surgical Equipment; Hormones; Mediastinum

\section{Introduction}

Hyperparathyroidism is the result of excessive production of parathyroid hormone from the parathyroid gland, and $15 \%$ to $20 \%$ abnormal parathyroid glands are ectopic. Ectopic parathyroid adenoma is a frequent cause of persistent and recurrent hyperparathyroidism, and $1 \%$ to $2 \%$ parathyroid adenomas are located in the mediastinum. Primary hyperparathyroidism caused by an ectopic mediastinal parathyroid adenoma is very rare.

The excision of ectopic mediastinal parathyroid adenoma can be difficult. Most mediastinal parathyroid adenomas are excised by surgery through a median sternotomy or thoracotomy, which may be associated with major morbidity [1,2]. Video-assisted thoracoscopic surgery is feasible and safe [3]. Robotic mediastinal parathyroidectomy also has been reported previously [4].

Giant cell tumor of bone is a rare sequela of the direct effect of parathyroid hormone on bone, occurring in $4.5 \%$ patients with primary hyperparathyroidism. Giant cell tumors may cause extensive bone resorption in the mandible, ribs, pelvis, and clavicles. These tumors usually have no autonomous growth and occur in more advanced stages of bone disease associated with hyperparathyroidism [5]. Giant cell tumors may regress after treatment of hyperparathyroidism.

We treated a woman who had a maxillary giant cell tumor associated with an ectopic parathyroid adenoma in the anterior mediastinum. Excision of the mediastinal tumor was facilitated with robotic surgery.

\section{Case Report}

A 50-year-old woman presented with a diagnosis of maxillary giant cell tumor and primary hyperparathyroidism (Figure 1). She was presented with slowly growing mass in the maxilla. The concentration of serum calcium was $3.5 \mathrm{mmol} / \mathrm{l}$ (normal reference range, 2.12 $\mathrm{mmol} / \mathrm{l}$ to $2.52 \mathrm{mmol} / \mathrm{l}$ ) and the concentration of serum phosphate was $0.37 \mathrm{mmol} / \mathrm{l}$ (normal reference range, $0.81 \mathrm{mmol} / \mathrm{l}$ to $1.58 \mathrm{mmol} / \mathrm{l}$ ). Primary hyperparathyroidism was clinically suspected. The concentration of plasma parathyroid hormone was $70.5 \mathrm{pg} / \mathrm{ml}$ (normal reference range, 1.6 to $6.9 \mathrm{pg} / \mathrm{ml}$ ). At Computed tomography of the thorax with intravenous contrast showed a mildly enhanced, oval soft tissue nodule $(2.5 \times 1.5 \times 1.7 \mathrm{~cm})$ at the anterosuperior mediastinum (Figure 2(a)). Singlephoton emission computed tomography/computed tomography with technetium $99 \mathrm{~m}$ sestamibi showed increased uptake at the nodule in the anterior mediastinum (Figure 2(b)). The patient underwent incisional biopsy of maxillary mass. The histological examination revealed multinucleated giant cells in cellular fibrous stroma with areas of new bone formation mixed with hemorrhage. 


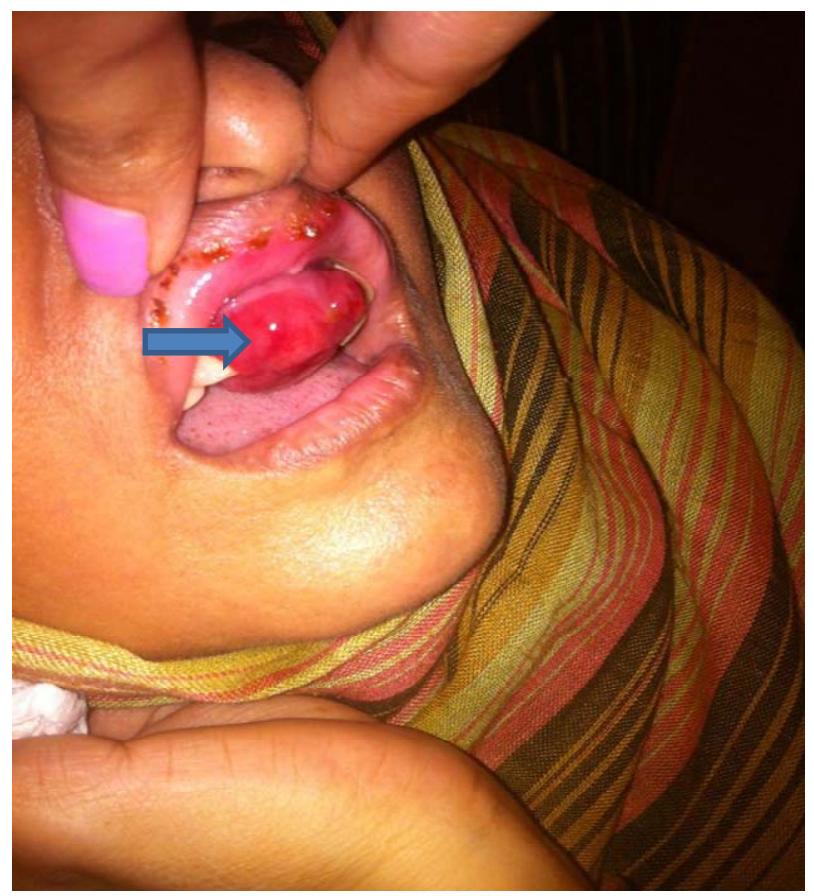

Figure 1. 50-year-old woman image shows a maxillary tumor (arrow).
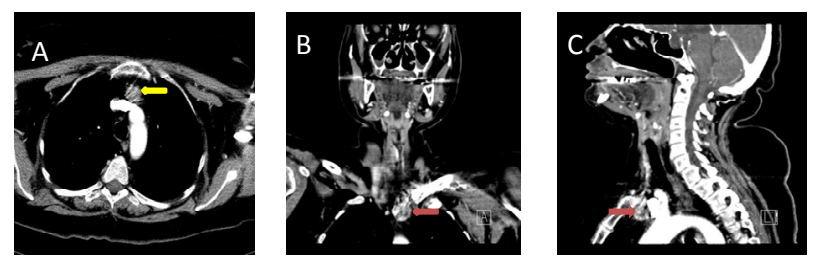

(a)

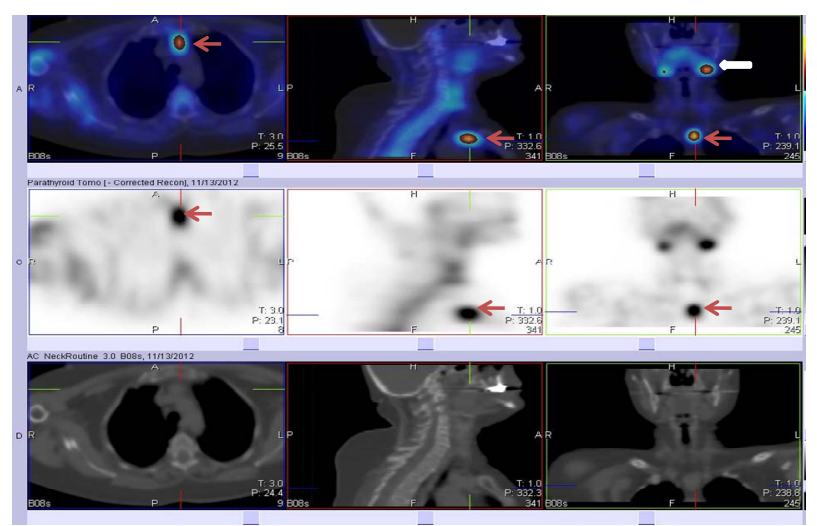

(b)

Figure 2. (a) Computed tomography scan of a 50-year-old woman who had a maxillary giant cell tumor and primary hyperparathyroidism. (A) Axial image shows ectopic parathyroid adenoma (yellow arrow) above the left brachiocephalic vein. $(B, C)$ Coronal and sagittal images show the ectopic parathyroid adenoma (red arrows); (b) Singlephoton emission computed tomography/computed tomography scan with technetium $99 \mathrm{~m}$ sestamibi shows increased uptake at the parathyroid adenoma (red arrows) and left submandibular salivary gland (white arrow).
These features are consistent with reparative giant cell granuloma.

Mediastinal parathyroidectomy was performed under general anesthesia with a 3-arm surgical robot (da Vinci Surgical System, Intuitive Surgical Inc., Sunnyvale, CA). A double-lumen endobronchial tube was placed for single lung ventilation, and the patient was positioned supine with a pillow under the left chest to tilt the left side up $30^{\circ}$. After 3 skin incisions were made along the left submammary fold, the camera port ( $30^{\circ}$ lens) was introduced through the fifth intercostal space along the ante rior axillary line. The right robotic arm was introduced through the fifth intercostal space at the midclavicular line and the left robotic arm was introduced through the third intercostal space, anterior to the midaxillary line. The left phrenic nerve was the reference landmark for the anterior mediastinum. The left arm held a grasper (EndoWrist precise grasper, Intuitive Surgical Inc., Sunnyvale, CA) and the right arm held a dissector (EndoWrist harmonic shear dissector, Intuitive Surgical Inc., Sunnyvale, CA). After the robotic system was engaged, dissecttion was performed along the left phrenic nerve to the jugular fold and inferiorly along the retrosternal region. The nodule was identified above the aortic arch and below the brachiocephalic artery. The dissection was continued along the left brachiocephalic vein, and feeding vessels from the brachiocephalic vein were clipped and divided. The nodule (2.6 g) was successfully removed, both frozen and permanent section histology confirmed hypercellular parathyroid adenoma. A chest tube was placed into the left pleural cavity at the completion of surgery and removed on the first postoperative day.

The postoperative course was uneventful and the patient was discharged home on the fourth postoperative day. After surgery, the plasma parathyroid hormone level decreased markedly (before surgery, 70.5 pg/ml; Day 1 after surgery, $5.5 \mathrm{pg} / \mathrm{ml}$; normal reference range, 1.6 to $6.9 \mathrm{pg} / \mathrm{ml})$.

During follow-up, the maxillay mass regressed significantly and the patient parathyroid hormone and serum calcium were normalized.

\section{Discussion}

The patient had a mediastinal ectopic parathyroid adenoma that was successfully excised with robotic surgery. The current robot-assisted technique provided several advantages, including markedly decreased morbidity compared with median sternotomy or thoracotomy. Furthermore, the corner of the anterior mediastinum, where this tumor was located, may be difficult to reach with video-assisted thoracoscopy. In addition, the 3-dimensional visualization, high-dexterity, and full range of motion of the robotic system provided an efficient and safe surgical procedure for this mediastinal tumor. The first 
robotic mediastinal parathyroidectomy was reported by Bonder et al. in 2004 [4]. We adopt the left-sided approach and the use of pneumomediastinum to facilitate dissection and improve visualization of the aortopulmonary window.

Giant cell tumors as a manifestation of primary hyperparathyroidism are extremely rare; usually these tumors have no autonomous growth and occur only in the more advanced stages of hyperparathyroidism bone disease [5]. After appropriate treatment of hyperparathyroidism, giant cell tumors tend to regress. We believe that robotic assisted parathyroidectomy is safe, feasible and preferable expedient alternative technique to open resection in the context of mediastinal pathology. Long term follow-up is warranted for maxillary tumor recurrence.

\section{REFERENCES}

[1] C. F. Russell, A. J. Edis, D. A. Scholz, P. F. Sheedy and J. A. van Heerden, "Mediastinal Parathyroid Tumors: Experience with 38 Tumors Requiring Mediastinotomy for Removal," Annals of Surgery, Vol. 193, No. 6, 1981, pp.
805-809. doi:10.1097/00000658-198106000-00016

[2] K. Cupisti, C. Dotzenrath, D. Simon, H. D. Röher and P. E. Goretzki, "Therapy of Suspected Intrathoracic Parathyroid Adenomas. Experiences Using Open Transthoracic Approach and Video-Assisted Thoracoscopic Surgery," Langenbeck's Archives of Surgery, Vol. 386, No. 7, 2002, pp. 488-493. doi:10.1007/s00423-001-0254-X

[3] R. J. Landreneau, S. R. Hazelrigg, P. F. Ferson, et al., "Thoracoscopic Resection of 85 Pulmonary Lesions," The Annals of Thoracic Surgery, Vol. 54, No. 3, 1992, pp. 415420. doi:10.1016/0003-4975(92)90430-C

[4] J. Bonder, C. Profanter, R. Prommegger, A. Greiner, R. Margreiter and T. Schmid, "Mediastinal Parathyroidectomy with the da Vinci Robot: Presentation of New Technique,” The Journal of Thoracic and Cardiovascular Surgery, Vol. 127, No. 6, 2004, pp. 1831-1832. doi:10.1016/j.jtcvs.2003.12.036

[5] Z. Meng, M. Zhu, Q. He, et al., "Clinical Implications of Brown Tumor Uptake in Whole-Body 99mTc-Sesta-Mibi Scans for Primary Hyperparathyroidism,” Nuclear Medicine Communications, Vol. 32, 2011, pp. 708-715. doi:10.1097/MNM.0b013e328347b582 